\title{
A field-deployable, chemical ionization time-of-flight mass spectrometer
}

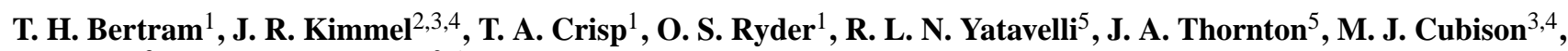 \\ M. Gonin ${ }^{3}$, and D. R. Worsnop ${ }^{2,6}$ \\ ${ }^{1}$ Department of Chemistry, University of California San Diego, La Jolla, CA, USA \\ ${ }^{2}$ Aerodyne Research Incorporated, Billerica, MA, USA \\ ${ }^{3}$ Tofwerk AG, Thun, Switzerland \\ ${ }^{4}$ Cooperative Institute for Research in Environmental Sciences, University of Colorado, Boulder, CO, USA \\ ${ }^{5}$ Department of Atmospheric Sciences, University of Washington, Seattle, WA, USA \\ ${ }^{6}$ Department of Physics, University of Helsinki, Helsinki, Finland
}

Received: 7 March 2011 - Published in Atmos. Meas. Tech. Discuss.: 22 March 2011

Revised: 7 July 2011 - Accepted: 8 July 2011 - Published: 20 July 2011

\begin{abstract}
We constructed a new chemical ionization timeof-flight mass spectrometer (CI-TOFMS) that measures atmospheric trace gases in real time with high sensitivity. We apply the technique to the measurement of formic acid via negative-ion proton transfer, using acetate as the reagent ion. A novel high pressure interface, incorporating two RF-only quadrupoles is used to efficiently focus ions through four stages of differential pumping before analysis with a compact TOFMS. The high ion-duty cycle $(>20 \%)$ of the TOFMS combined with the efficient production and transmission of ions in the high pressure interface results in a highly sensitive ( $>300$ ions s ${ }^{-1}$ pptv $^{-1}$ formic acid) instrument capable of measuring and saving complete mass spectra at rates faster than $10 \mathrm{~Hz}$. We demonstrate the efficient transfer and detection of both bare ions and ion-molecule clusters, and characterize the instrument during field measurements aboard the $R / V$ Atlantis as part of the CalNex campaign during the spring of 2010. The in-field short-term precision is better than $5 \%$ at $1 \mathrm{pptv}(\mathrm{pL} / \mathrm{L})$, for $1-\mathrm{s}$ averages. The detection limit ( $3 \sigma, 1$-s averages) of the current version of the CITOFMS, as applied to the in situ detection of formic acid, is limited by the magnitude and variability in the background determination and was determined to be 4 pptv. Application of the CI-TOFMS to the detection of other inorganic and organic acids, as well as the use of different reagent ion molecules (e.g. $\mathrm{I}^{-}, \mathrm{CF}_{3} \mathrm{O}^{-}, \mathrm{CO}_{3}^{-}$) is promising, as we have demonstrated efficient transmission and detection of both bare ions and their associated ion-molecule clusters.
\end{abstract}

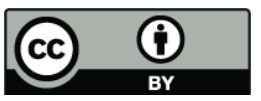

Correspondence to: T. H. Bertram (thbertram@ucsd.edu)

\section{Introduction}

The atmospheric measurement of reactive trace gases pose unique instrumental challenges that differ significantly from those found in the laboratory (Farmer and Jimenez, 2010). Selective detection at trace levels necessitates high sensitivity with response factors that are robust against dramatic changes in sampling conditions (e.g. temperature, relative humidity, and trace gas and aerosol loadings). Further, instrument physical size and power consumption must be minimized for deployment aboard research platforms with limited space and power. In recent years, chemical ionization mass spectrometry (CI-MS) has been successfully applied to the sensitive, selective, and accurate detection of a large suite of trace gases from a variety of sampling platforms (Huey, 2007). CI-MS detection techniques permit the simultaneous observation of a variety of compounds determined by the choice of reagent ion, where the specificity of the ionization process is dependent on the ion-molecule reaction mechanism. For example, $\mathrm{SF}_{6}^{-}$has been routinely used as a generic reagent ion, where analyte ions are formed following either charge transfer or fluorine adduct formation (Huey et al., 1995). In addition to the suite of general reagent ions that are used extensively in the laboratory, there is great interest in the atmospheric community in the development of selective chemical ionization methods that target specific classes of molecules (Morrison and Howard, 2001; Slusher et al., 2001; Veres et al., 2008).

At present, the majority of field-deployable CI-MS instruments are designed around a quadrupole mass spectrometer (QMS), where near unit ion transmission in the quadrupole is highly advantageous for detection of trace compounds.

Published by Copernicus Publications on behalf of the European Geosciences Union. 
Detection thresholds of less than two ppqv (5-min averages) have been achieved with atmospheric pressure/chemical ionization mass spectrometers (Berresheim et al., 2000). Typical sampling routines consist of tuning the quadrupole mass filter sequentially through a series of selected mass-to-charge ratios $(\mathrm{m} / \mathrm{Q})$, where the dwell time at each $\mathrm{m} / Q$ value is of order $0.1 \mathrm{~s}$. This routine is often punctuated by intermittent scans over the full range of mass-to-charge ratios accessible by the QMS configuration. As the number of monitored values increases, the sampling duty cycle for each individual component decreases, resulting in a corresponding increase in the limit of detection. The scanning procedure impacts data coverage and causes a misalignment in both time and space for data corresponding to different $m / Q$ values obtained from instruments on mobile platforms. When sampling from mobile platforms or when attempting quasicontinuous measurements used in analyses such as eddy covariance, researchers often monitor only a single or very small number of compounds, in order to maintain spatial and/or temporal continuity in data. Beyond this, typical CIQMS instruments used for atmospheric measurements are unable to resolve ions having the same nominal mass, making analysis of complex mass spectra challenging. To address these issues, recent instrumental development has been focused on the coupling of high-pressure chemical ionization sources with time-of-flight (TOF) (Blake et al., 2004, 2006; Ennis et al., 2005; Graus et al., 2010; Jordan et al., 2009; Tanimoto et al., 2007; Wyche et al., 2007) and ion trap (Mielke et al., 2008; Müller et al., 2009; Prazeller et al., 2003; Steeghs et al., 2007; Warneke et al., 2005) mass spectrometers. The higher resolving power and improved sample coverage (i.e. complete mass spectra at $\mathrm{kHz}$ sampling rates) achieved with TOF mass spectrometers make TOF-based CI-MS instruments particularly well suited for atmospheric measurements of trace gases. To a first approximation, the relative sensitivity of QMS and TOFMS based instruments using the same ion source and interface optics is the ratio of their respective duty cycles (DeCarlo et al., 2006; Guilhaus et al., 2000). Where-as the sampling duty cycle of a QMS is inversely proportional to the number of $\mathrm{m} / \mathrm{Q}$ values monitored, the duty cycle of an orthogonal extraction (OE) TOFMS (as used in this work) is inversely proportional to the extraction frequency (or the square root of the maximum $m / Q$ value monitored). Typical OETOFMS sampling duty cycles are in the range of 10 to $50 \%$, and, for fixed conditions, the value increases as the square root of the mass-to-charge ratio. For experiments aiming to measure the concentration and $m / Q$ values of a large number of different substances, the TOFMS has clear advantages in terms of both data continuity and sampling duty cycle. Additionally, the use of reagent ions capable of ionizing a specific class of compounds, such as acids (Veres et al., 2008), enables the simultaneous detection of a broad range of compounds, further exploiting this advantage.
This paper characterizes a high-pressure chemical ionization time-of-flight mass spectrometer (CI-TOFMS), which was built by adapting a well-known flow-tube CI source (Kercher et al., 2009) with a commercial atmosphericpressure interface compact TOFMS (API-CTOF, Tofwerk AG, Thun, Switzerland) (Junninen et al., 2010). We demonstrate that the newly developed CI-TOFMS has the sensitivity, accuracy, and precision required for atmospheric observations from a wide array of measurement platforms. In the following manuscript we assess the performance of the CI-TOFMS configured as a negative-ion proton-transfer mass spectrometer, using acetate ion chemistry (Veres et al., 2008), however a number of different negative (e.g. $\mathrm{I}^{-}$, $\mathrm{CF}_{3} \mathrm{O}^{-}, \mathrm{SF}_{6}^{-}$) or positive (e.g. $\mathrm{H}_{3} \mathrm{O}^{+}, \mathrm{NO}^{+}$) reagent ions (Hearn and Smith, 2004) could easily be employed using similar hardware.

\section{Experimental section}

\subsection{Instrument description}

The CI-TOFMS consists of five differentially pumped stages as shown in Fig. 1. Here, ambient air is sampled through a critical orifice at $1.51 \mathrm{~min}^{-1}$ into the ion-molecule reaction (IMR) chamber. The first section of the stainless steel reaction chamber is $2.5 \mathrm{~cm}$ long and has an inner diameter (ID) of $3.5 \mathrm{~cm}$. The second section of the IMR chamber is $5 \mathrm{~cm}$ in length, and is linearly tapered to reduce the ID to $0.6 \mathrm{~cm}$ at the exit. The IMR chamber is pumped by a $1001 \mathrm{~min}^{-1}$ dry scroll pump (Varian SH-110), that is typically throttled down to achieve a sampling pressure of $85 \mathrm{mbar}$, however diagnostic experiments have been run in the pressure range of 20 130 mbar. Reagent ions are introduced into the IMR chamber orthogonal to the sample flow at a flow rate of $2.01 \mathrm{~min}^{-1}$, approximately $2 \mathrm{~cm}$ from the entrance orifice. In this application, acetate ions $\left(\mathrm{CH}_{3} \mathrm{C}(\mathrm{O}) \mathrm{O}^{-}\right)$are formed by passing trace acetic anhydride in ultra high purity (UHP) nitrogen $\left(\mathrm{N}_{2}, 99.9995 \%\right)$ through a commercial ${ }^{210} \mathrm{Po}$ alpha emitter (NRD, P-2021 EOL Ionizer), following the initial work of Veres et al. (2008). Under typical sampling conditions, the ion-molecule reaction time is of order $0.1 \mathrm{~s}$ in the IMR chamber. The entire IMR chamber, including the ionizer, is electrically isolated and biased to $-100 \mathrm{~V}$ to facilitate efficient transport of ions to the entrance aperture of the second pumping stage.

The IMR is subsampled through a second critical orifice $(500 \mu \mathrm{m})$ into the collisional dissociation chamber (CDC), which contains a short, segmented RF-only quadrupole having tunable frequency and amplitude. The segmented quadrupole, consisting of six resistively coupled segments, permits creation of an electric field within the CDC for effective collisional dissociation of weakly bound ion-molecule clusters, while simultaneously focusing and cooling the expansive beam entering from the IMR. The CDC entrance 


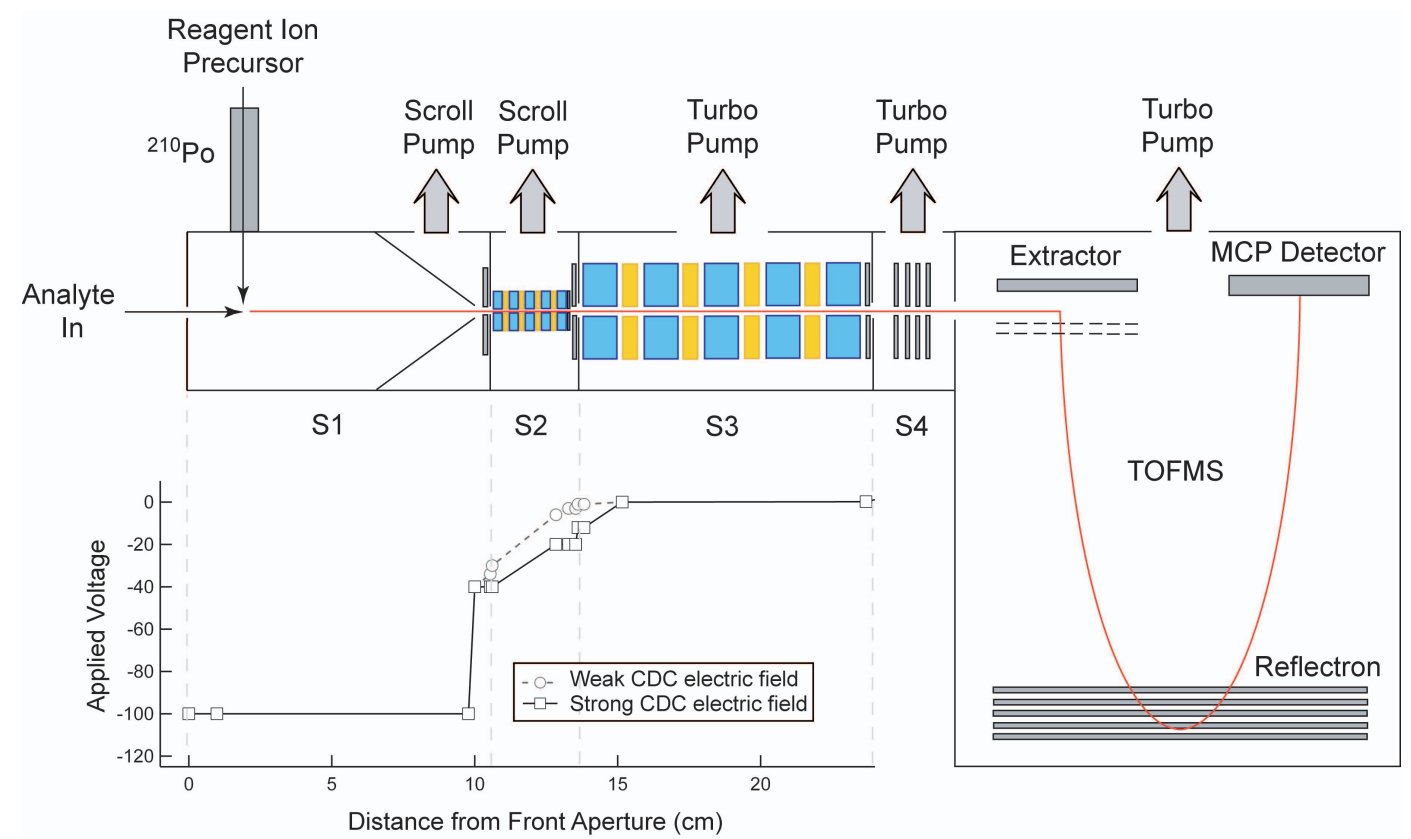

Fig. 1. Schematic of the chemical ionization source and four stage (S1-S4) differentially pumped interface coupled to the time-of-flight mass spectrometer (TOFMS, $5 \times 10^{-7} \mathrm{mbar}$ ). The high pressure interface consists of: (i) ion-molecule reaction (IMR) chamber (S1, 85 mbar), (ii) collisional dissociation chamber (CDC) (S2, 2 mbar), (iii) stage 3 that houses a second RF-only segmented quadrupole (S3, $1.5 \times 10^{-2} \mathrm{mbar}$ ), and (iv) stage 4 that houses a series of DC optics that focus and accelerate the primary beam into the TOFMS (S4, $3.5 \times 10^{-5} \mathrm{mbar}$ ). DC voltages applied to the focusing electrodes under two different declustering conditions, are shown in the inset figure, as a function of the distance from the entrance aperture.

aperture is electrically biased to $-40 \mathrm{~V}$ and followed by a weak electric field along the axis of the CDC quadrupole. The CDC electric field can be tuned to either transmit or dissociate weakly bound clusters with less than $10 \%$ impact on the total ion throughput, as discussed below. The CDC pressure is controlled to 2 mbar by a $2501 \mathrm{~min}^{-1}$ dry scroll pump (Varian TriScroll 300) that also serves as a backing pump for the split-flow turbo pump used in the downstream, low-pressure stages. The focused ion beam exits the CDC through a $1 \mathrm{~mm}$, electrically biased aperture into a second RF-only quadrupole, housed at $1.5 \times 10^{-2}$ mbar. The ion beam is then directed into the fourth stage of differential pumping, held at $3.5 \times 10^{-5} \mathrm{mbar}$, which houses a series of DC optics that focus and accelerate the primary beam into the orthogonal extraction, reflectron TOFMS $\left(5.0 \times 10^{-7} \mathrm{mbar}\right)$. The interface stages tailor the spatial and energetic profile of the primary ion beam to match the requirements of the OETOFMS. This work uses a compact TOFMS to achieve high sensitivity; the same ion source and interface is also suitable for higher resolution TOFMS platforms (Junninen et al., 2010). The second quadrupole chamber, the DC optics chamber, and the TOFMS are pumped by three isolated stages of a split flow turbo pump (Pfeiffer), with speeds of 20, 155 , and $2001 \mathrm{~s}^{-1}$, respectively. The compact TOFMS has an effective ion path length of approximately $0.5 \mathrm{~m}$, and is configured for analysis of positive or negative ions. Typical extraction frequencies in this work were on the order of $15 \mu \mathrm{s}(66 \mathrm{kHz})$, corresponding to a recorded $m / Q$ range of 0-200 thomson (Th, $1 \mathrm{Th}=1 \mathrm{u} \mathrm{e}^{-1}$ ). In its current configuration, the CI-TOFMS, excluding the two scroll pumps and power supplies, is contained within a $50 \mathrm{~cm} \times 40 \mathrm{~cm} \times 40 \mathrm{~cm}$ frame, permitting it to be deployed to a wide range of sampling platforms.

\subsection{Data acquisition and signal quantification}

TOFMS timing, data acquisition, and data storage are controlled by the Tofwerk TofDaq software package running on a dual-core PC (Dell Precision R5400 Rack Workstation, 2 GB RAM, RAID0 disk). The output of the microchannel plate (MCP) detector of the TOFMS is passed to an 8-bit PCI analog-digital converter (ADC, Acqiris AP240, Agilent, Geneva, Switzerland), which digitizes and records data on two channels at $1 \mathrm{GS} \mathrm{s}^{-1}$. Signals recorded on the first channel are amplified by a factor of 11 upstream of the ADC; the second channel records the un-amplified MCP output. The offsets of the two channels are adjusted so that the second channel records only those portions of the signal that exceed the full scale of the first. The TofDaq acquisition software sums the signal waveforms from the two channels, with normalization for the difference in amplification. This two-channel procedure extends the dynamic range of the 8-bit ADC, and is necessary to quantify the large reagent 
ion signals observed in this system without compensating the system's ability to accurately record low intensity signals. Accurate recording of small signals is further enhanced by the noise-suppression-accumulation functionality of the AP240 ADC, which discards digitized samples having intensity below a user-defined threshold, prior to averaging of data in the ADC memory.

Areas of ion peaks in digitized mass spectra are converted to units of ions $\mathrm{s}^{-1}$ by normalization, using a value representing the TOFMS response to single-ion detection events. To measure this value, data are recorded with the TOFMS extraction electrodes held at $0 \mathrm{~V}$. Under these conditions, the MCP detector detects randomly scattered ions at a low rate. Ion events are distinguished from electronic noise based on a user-adjusted threshold. An average single-ion detection peak shape is established based on a collection of recorded events (typically thousands), and the area of this "single ion signal" is used for calibrating the intensity of MS data. Data are saved as Hierarchical Data Format Version 5 (HDF5) files (HDF Group, Champaign, IL, www.hdfgroup.org). For each averaged MS written to disk, two data structures are stored: a time-of-flight spectrum having units of accumulated signal intensity versus ion time-of-flight and a list of summed areas for user-defined regions of the mass spectrum, called "peak data". The TOF spectrum can be calibrated in post processing and used for exact mass analysis and/or peak integration, whereas the peak data contains the result of the peak integration, based on the mass calibration established at the time the data were recorded. In this work, peak data summation regions were centered on integer $m / Q$ values, with width sufficient to capture all ions of equal nominal mass. For the remainder of this manuscript we report ion count rates as "unit mass resolution" (UMR) peak areas. Fitting peaks in the mass-calibrated TOF spectrum allows determination of exact $m / Q$, whereas integration to UMR peaks (as illustrated in Fig. 3) enables quantification, assuming no interferences.

\subsection{Ion chemistry}

Here, we report the application of the CI-TOFMS toward the selective detection of formic acid via negative-ion proton transfer, using acetate as the reagent ion. We note that acetate ion chemistry has been shown to be effective in the selective detection of a wide array of both organic and inorganic acids (Roberts et al., 2010), and while not specifically addressed here, it is expected that the CI-TOFMS described here has the capability to measure other acids as well. Acetate ions have been shown to react selectively with acids having a gas-phase acidity larger than that of acetic acid $\left(\Delta G=341.5 \mathrm{kcal} \mathrm{mol}^{-1}\right)$ (Veres et al., 2008). As discussed in Graul et al. (1990) the ion-molecule reaction proceeds through either proton transfer from the target acid to the reagent ion (Reaction R1) or adduct formation (Reaction R2).

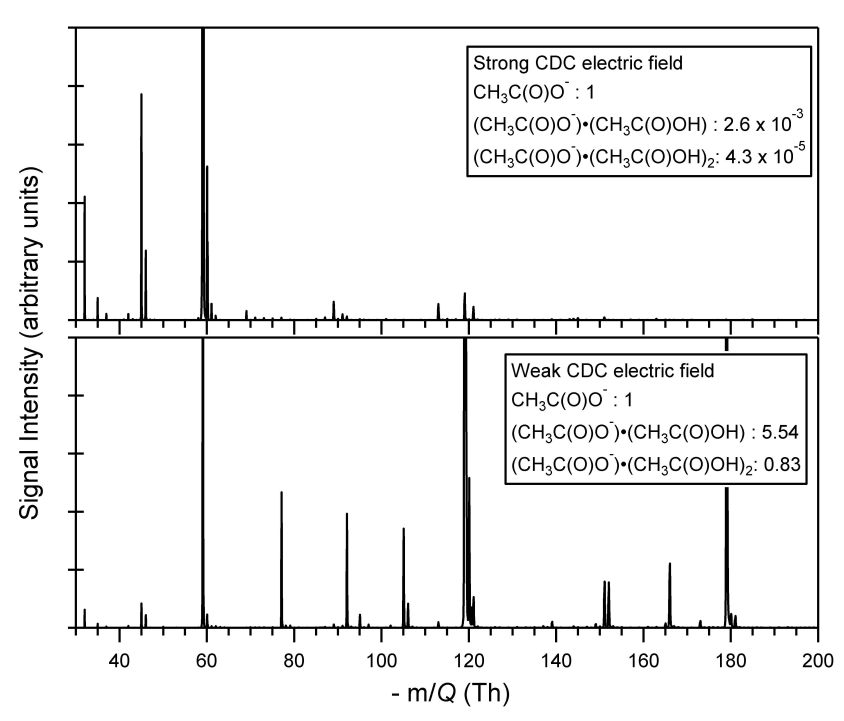

Fig. 2. Sample mass spectrum taken at $2 \mathrm{~Hz}$ on ambient air using the two declustering schemes shown in Fig. 1b. In a strong CDC electric field, acetate ions are primarily observed as bare ions (top), while in a weak $\mathrm{CDC}$ electric field acetate can be observed in significant quantities in both the $\left(\mathrm{CH}_{3} \mathrm{C}(\mathrm{O}) \mathrm{O}^{-}\right) \cdot\left(\mathrm{CH}_{3} \mathrm{C}(\mathrm{O}) \mathrm{OH}\right)$ cluster $(-119.034 \mathrm{Th})$ and the $\left(\mathrm{CH}_{3} \mathrm{C}(\mathrm{O}) \mathrm{O}^{-}\right) \times\left(\mathrm{CH}_{3} \mathrm{C}(\mathrm{O}) \mathrm{OH}\right)_{2}$ cluster $(-179.055 \mathrm{Th})$ (bottom). The ratio of the cluster ion count rate to the bare ion count rate is shown in the figure legend.

$$
\begin{aligned}
& \mathrm{CH}_{3} \mathrm{C}(\mathrm{O}) \mathrm{O}^{-}+\mathrm{HX} \longrightarrow \mathrm{CH}_{3} \mathrm{C}(\mathrm{O}) \mathrm{OH}+\mathrm{X}^{-} \\
& \mathrm{CH}_{3} \mathrm{C}(\mathrm{O}) \mathrm{O}^{-}+\mathrm{HX} \longrightarrow \mathrm{CH}_{3} \mathrm{C}(\mathrm{O}) \mathrm{O}^{-}(\mathrm{HX})
\end{aligned}
$$

The branching ratio between these two product channels is likely dependent on the pressure and reaction time in the IMR region, however we note that under the sampling conditions used here $\left(P_{\mathrm{IMR}}=20-100 \mathrm{mbar}\right.$, $\left.P_{\mathrm{CDC}}=2 \mathrm{mbar}\right)$ the reaction proceeds predominately through Reaction (R2). At high average CDC electric field strengths $\left(-80 \mathrm{~V} \mathrm{~cm}^{-1}\right)$ collisional dissociation of the acetate-analyte clusters $\left.\left(\mathrm{CH}_{3} \mathrm{C}(\mathrm{O}) \mathrm{O}^{-}\right) \cdot \mathrm{HX}\right)$ results in the formation of the deprotonated acid $\left(\mathrm{X}^{-}\right)$. At low average electric field strength $\left(-15 \mathrm{~V} \mathrm{~cm}^{-1}\right)$, clusters are efficiently transferred through the CDC, before extraction into the TOFMS. It is most likely that the majority of the declustering occurs between the exit of the CDC quadrupole and the entrance aperture to the second quadrupole chamber, where the local electric field divided by the gas number density $(E / N)$ was greater than 400 Townsend $\left(\mathrm{Td}=10^{-17} \mathrm{~V} \mathrm{~cm}^{-2}\right)$ under high declustering conditions. Figure 1 depicts two different electric fields used in the CI-TOFMS for either efficient collisional dissociation (strong electric field) or cluster transmission (weak electric field). The resulting mass spectra, recorded at $2 \mathrm{~Hz}$ while sampling ambient air, for the two conditions are shown in Fig. 2. Under strong electric field strength, the vast majority of the detected reagent ions are observed as $\left(\mathrm{CH}_{3} \mathrm{C}(\mathrm{O}) \mathrm{O}^{-}\right)(-59.013 \mathrm{Th})$, with minimal contribution 


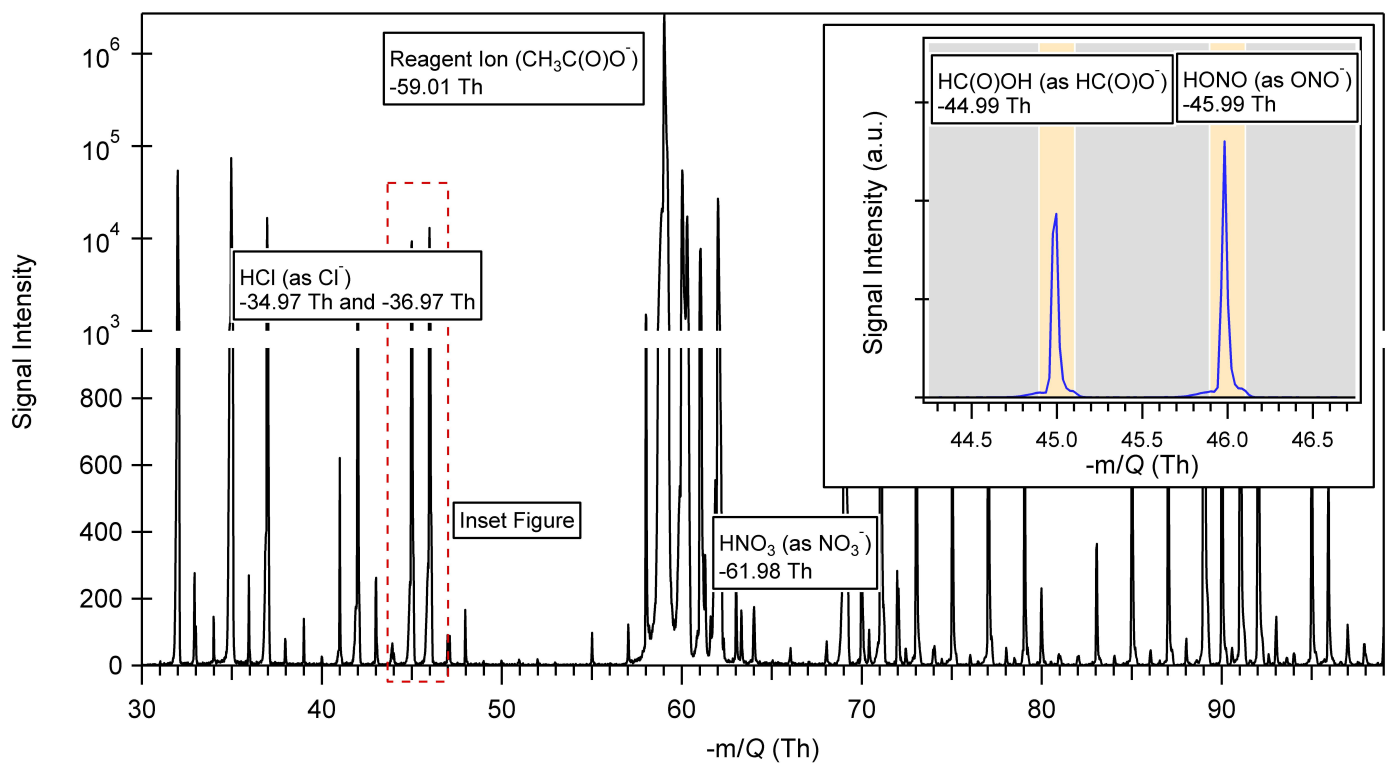

Fig. 3. Negative ion CI-TOFMS mass spectrum, acquired at $2 \mathrm{~Hz}$ using acetate $\left(\mathrm{CH}_{3} \mathrm{C}(\mathrm{O}) \mathrm{O}^{-}\right)$reagent ions. The spectrum was taken on ambient air measured aboard the $R / V$ Atlantis during the CalNex Campaign in May 2010. Inset figure Formic and nitrous acid peaks, observed at $-44.99 \mathrm{Th}$ and $-45.99 \mathrm{Th}$, respectively. The yellow shaded region represents the UMR peak integration window, and the grey shaded region the background determination window. The resolving power $(M / \Delta M)$ at $-44.99 \mathrm{Th}$ is $900 \mathrm{Th} \mathrm{Th}^{-1}$.

from higher order acetate-acetic acid clusters. In contrast, at weak electric field strength, reagent ions are observed primarily as $\left(\mathrm{CH}_{3} \mathrm{C}(\mathrm{O}) \mathrm{O}^{-}\right) \cdot\left(\mathrm{CH}_{3} \mathrm{C}(\mathrm{O}) \mathrm{OH}\right)(-119.034 \mathrm{Th})$, with substantial contributions from both the bare acetate ion and higher order clusters. It is important to note that the total ion count rate for reagent species $\left(3.0 \times 10^{7}\right.$ ions s $\left.^{-1}\right)$ in this experiment was conserved (to within $10 \%$ ) between the two choices of CDC electric field, demonstrating the efficient transfer, extraction, and detection of weakly bound ion-molecule clusters.

\subsection{Field operation}

The CI-TOFMS, operated as a negative ion proton transfer system, was deployed aboard the Research Vessel $(R / V) A t$ lantis as part of the CalNex field campaign in the spring of 2010. Data were collected for 25 days off the coast of California. In the following sections we describe the performance of the CI-TOFMS under operating conditions characteristic of atmospheric sampling, concentrating specifically on instrumental response to formic acid $(\mathrm{HC}(\mathrm{O}) \mathrm{OH})$. We assess the CI-TOFMS performance using formic acid, due to the availability of an accurate and stable calibration source. Ambient observations of the complete suite of detectable acids and a detailed discussion of inlet sampling conditions will be described in a second, separate manuscript that is in preparation. For the duration of the field campaign, MS data were acquired for the negative $m / Q$ range $10-200 \mathrm{Th}$ and saved at a rate of $2 \mathrm{~Hz}$. The system was operated at the pressures depicted in Fig. 1 and with a strong CDC electric field $\left(-80 \mathrm{~V} \mathrm{~cm}^{-1}\right)$. A representative ambient mass spectrum is shown in Fig. 3, where signal associated with hydrochloric, formic, nitrous, nitric, as well as a host of organic acids are readily apparent. Acquisition of the complete spectrum is advantageous for atmospheric observations, as it permits simultaneous measurement of many molecules, including those with unrecognized importance at the time of measurement. The acetate reagent ion count rate during CalNex, determined as UMR peak $-59 \mathrm{Th}$, was on average $3.0 \times 10^{7}$ ions s $^{-1}$. Detrimental ringing is not observed following the large reagent ion peak at $-59 \mathrm{Th}$. While the signal from this peak does have a measurable falling edge tail (scattered ions), it does not prevent us from observing ions at $-60 \mathrm{Th}$. The baseline count rate at $-60 \mathrm{Th}$, calculated from the tail of the reagent ion peak at $-59 \mathrm{Th}$, was less than 1000 ions s $^{-1}$, indicating that electronic or chemical noise associated with high reagent count rates has little impact on detection at adjacent masses.

\subsection{Mass resolving power and mass accuracy}

The mass resolving power of the compact CI-TOFMS, defined as $M / \Delta M$, where $\mathrm{M}$ is the peak center and $\Delta M$ is the full-width half-maximum of the fitted Gaussian peak, was greater than $900 \mathrm{Th} \mathrm{Th}^{-1}$ for the $m / Q$ range considered (10-200 Th). In the laboratory, a mass resolving power of $1100 \mathrm{Th} \mathrm{Th}^{-1}$ at $-200 \mathrm{Th}$ is routinely achieved, a result of continuous and more effective tuning. This slight improvement over the identical compact TOFMS operated with an electron impact (EI) ionization source (DeCarlo et 
al., 2006), is attributed to the less energetic beam generated by the high-pressure chemical ionization source and interface. As an example of what this resolving power enables, at $-45 \mathrm{Th}$, this instrument can resolve ions differing by more $0.04 \mathrm{Th}(45 \mathrm{Th} / 1100)$. It cannot, for instance, resolve the deprotonated carbon-13 isotopologue of formic acid $(-46.001 \mathrm{Th})$ and nitrous acid $(-45.993 \mathrm{Th})$, which differ by only $0.008 \mathrm{Th}$. As discussed earlier, this work chose the compact TOF platform for its speed and sensitivity. The same design can easily be adapted for use with the Tofwerk HTOF platform, which has higher resolution but lower sensitivity. The mass accuracy was determined as the deviation in the observed peak center from the expected exact mass for a series of peaks not used in the mass calibration. The relative mass accuracy for data obtained during CalNex was less than $0.1 \mathrm{mTh} \mathrm{Th}^{-1}(<100 \mathrm{ppm})$.

\section{Results and discussion}

\subsection{Sensitivity and absolute accuracy}

The sensitivity of the CI-TOFMS to formic acid was assessed by direct calibration of the instrument response to known concentrations of formic acid carried in UHP zero air. Atmospherically relevant concentrations of formic acid $(0-10 \mathrm{ppbv})$ were produced by mixing UHP zero air with $10 \mathrm{~cm}^{3} \min ^{-1} \mathrm{~N}_{2}$ that was passed over a gravimetrically calibrated permeation tube (Kin-Tek, SRT-2, $21.6 \mathrm{ng} \mathrm{min}^{-1}$ at $50^{\circ} \mathrm{C}$ ). The permeation tube, housed in a PFA Teflon tube, was contained in an insulated aluminum block, temperature controlled to $50 \pm 0.1^{\circ} \mathrm{C}$ by a PID temperature controller (Omega, model CNi16). The absolute accuracy of the CITOFMS technique is driven primarily by uncertainty in the emission rate of the permeation tube standard. We estimate, conservatively, that this is $20 \%$, based on ion chromatography analysis. Instrument response to formic acid was measured as the UMR peak at $-45 \mathrm{Th}$, and was observed to be a linear function of the delivered formic acid concentration up to approximately $1 \mathrm{ppbv}$, where titration of the acetate reagent ions led to decreased sensitivity. The observed count rate at $-45 \mathrm{Th}$ was normalized by the ratio of the reagent ion count rate in the absence of formic acid to that observed at each calibration step. The normalized sensitivity of the CI-TOFMS towards formic acid, as shown in Fig. 4, was observed to be 338 ions s${ }^{-1} \mathrm{pptv}^{-1}$. Similar to Veres et al. (2008) we do not observe a humidity dependence in the sensitivity. In Fig. 4, both the measured and normalized count rate at $-45 \mathrm{Th}$ and the reagent ion count rate at $-59 \mathrm{Th}$ are shown as a function of the formic acid concentration.

\subsection{Limit of detection}

The detection limit of the CI-TOFMS is a strong function of the magnitude and variability in the instrument zero. In the

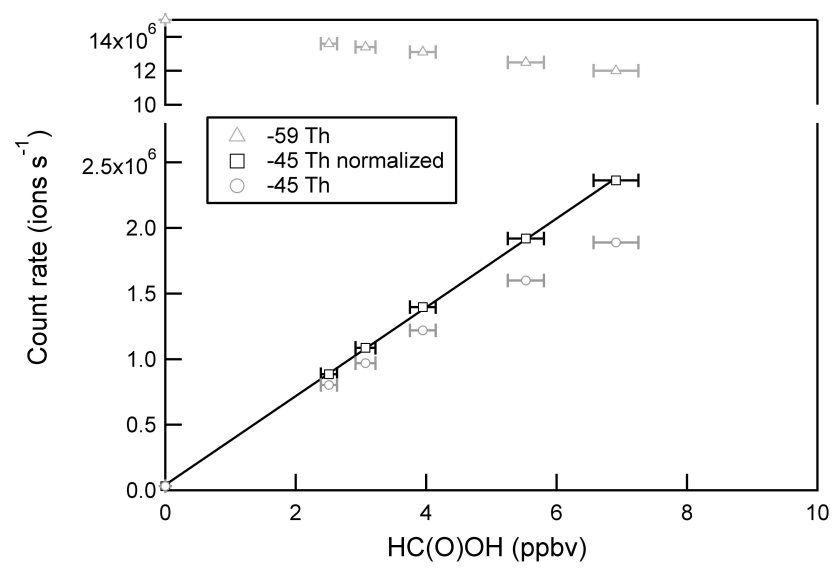

Fig. 4. Normalized calibration to formic acid (detected as $\mathrm{HC}(\mathrm{O}) \mathrm{O}^{-}$at $-45 \mathrm{Th}$ ). Both the measured (gray circles) and normalized (black squares) count rate at $-45 \mathrm{Th}$ and the reagent ion count rate at $-59 \mathrm{Th}$ (gray triangles) are shown as a function of the formic acid concentration.

following section, we assess the detection limit during routine atmospheric sampling for the field deployable system as operated aboard the $R / V$ Atlantis during CalNex. The CITOFMS response at $-45 \mathrm{Th}$, while sampling UHP zero air, was routinely $3 \times 10^{4}$ ions s $^{-1}$ (100 pptv). The high background concentration is attributed to a steady flux of formic acid from the inlet manifold, and can be reduced to less than $3 \times 10^{3}$ ions s$^{-1}$ (10 pptv) in laboratory measurements. The background count rate for peaks not associated with trace acids is routinely observed to be less than 10 ions s$^{-1}$, indicating that the formic acid background is not related to electronic noise in the TOFMS.

Assuming that random uncertainty in the observed counts follows Poisson statistics, the uncertainty in the signal and background count rate should be equivalent to the square root of the count rate, and thus the signal-to-noise ratio can be expressed as:

$$
\frac{S}{N}=\frac{C_{\mathrm{f}}[X] t}{\sqrt{C_{\mathrm{f}}[X] t+2 B t}}
$$

where $B$ is the background count rate, $C_{\mathrm{f}}$ is the calibration factor, $[X]$ is the mixing ratio, and $t$ is the integration time. Using the measured calibration factor of 338 ions s${ }^{-1} \mathrm{pptv}^{-1}$, and a background count rate of $3 \times 10^{4}$ ions s $^{-1}$, we calculate a detection limit of $3 \mathrm{pptv}$, for 1-s averaging, for a signal-tonoise ratio of 3 .

However, routine measurements of the background count rate during CalNex indicate that uncertainty in the background is not solely a function of Poisson statistics, as the background count rate at $-45 \mathrm{Th}$ drifts in time. To assess the impact of time dependent fluctuations in the background count rate, we can calculate the detection limit as the $3 \sigma$ uncertainty of the zero determinations. As an upper limit to the variability in the background determination, and ultimately 


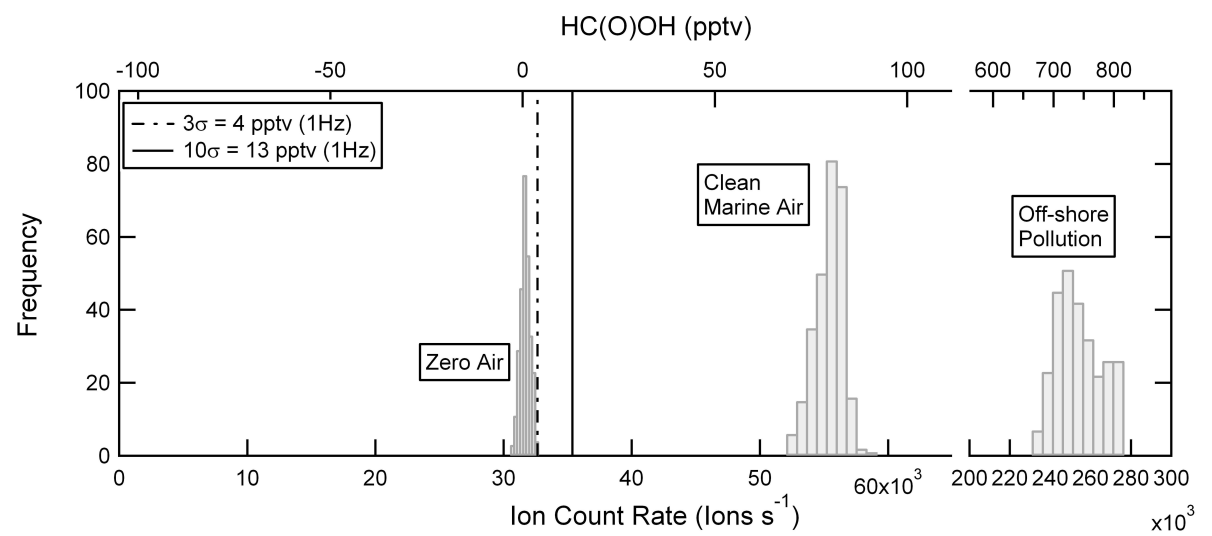

Fig. 5. Frequency distributions of ion count rate at $-45 \mathrm{Th}$ of $1 \mathrm{~Hz}$ data taken from $5 \mathrm{~min}$ sampling periods while sampling zero air, clean marine air, and polluted air aboard the $R / V$ Atlantis.

the calculated detection limit, we report the $3 \sigma$ uncertainty of $23 \mathrm{pptv}$ for the collection of all background determinations made over the course of a $24 \mathrm{~h}$ sampling period. In most cases, uncertainty in the background count rate varies slowly in time and an accurate representation of the uncertainty can be taken as the $3 \sigma$ uncertainty of successive zero determinations. To illustrate this point, we sample UHP zero air for $5 \mathrm{~min}$ and calculate the $3 \sigma$ uncertainty in the zero determination, from a Gaussian fit to the observations. At the background count rate measured here $\left(3 \times 10^{4}\right.$ ions s $\left.^{-1}\right)$, the variability in the background can be assessed using Gaussian statistics, as there is little difference between Gaussian and Poisson statistics at high count rates. For measurements made during CalNex, we calculate a $3 \sigma$ and $10 \sigma$ value of $4 \mathrm{pptv}$ and $13 \mathrm{pptv}$, respectively (for 1 -s averages). The $3 \sigma$ value of 4 pptv is representative of our measurements during CalNex, and indicates that if we were to characterize the background at five-minute intervals during field observations, a detection limit of 4 pptv (with 1-s averaging) can be achieved. For comparison, the distribution of measured count rates for five minutes of sampling in both remote and polluted air masses are shown in Fig. 5, indicating that the detection limit is well below the atmospheric abundance of formic acid even in pristine conditions. The concentrations reported in Fig. 5, for clean marine air (50-100 pptv) and offshore pollution (650-850 pptv) are within the range of values reported in the literature for measurement of formic acid concentrations in urban areas (1-10 ppbv) and remote continental and marine areas ( $<1$ ppbv) (Grosjean et al., 1990; Keene et al., 1989; Paulot et al., 2011; Veres et al., 2008). At present, the formic acid detection limit is limited by the magnitude and variability in the background. We are focusing our current efforts on reducing this and expect that future versions of the instrument will have significantly improved detection limits as a result.

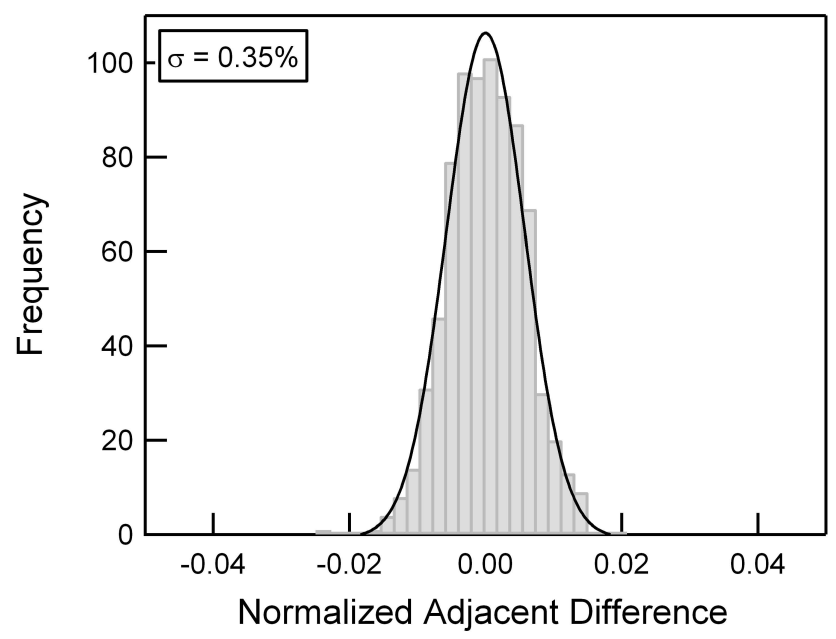

Fig. 6. Distribution of normalized adjacent differences, as measured on a formic acid concentration of $2.3 \mathrm{ppbv}$ at $1 \mathrm{~Hz}$, yielding an upper limit to the instrument precision $(1 \sigma)$ of $0.35 \%$ at $2.3 \mathrm{ppbv}$.

\subsection{Short and long term precision}

To assess the short-term precision of the CI-TOFMS, we calculate the normalized difference between adjacent points for 15 min of measurement at $1 \mathrm{~Hz}$, at a constant formic acid concentration via Eq. (2).

$\mathrm{NAD}=\frac{[X]_{n}-[X]_{n-1}}{\sqrt{[X]_{n}[X]_{n-1}}}$

The standard deviation of the Gaussian fit to the normalized distribution of the normalized adjacent differences (NAD) is a direct measure of the instrument precision (Fig. 6). From this analysis, the upper limit to the short-term precision $(1 \sigma)$ is $0.35 \%$ for a formic acid concentration of $2.3 \mathrm{ppbv}$. The short-term precision, derived from the normalized adjacent difference analysis, was calculated for a range of count rates 


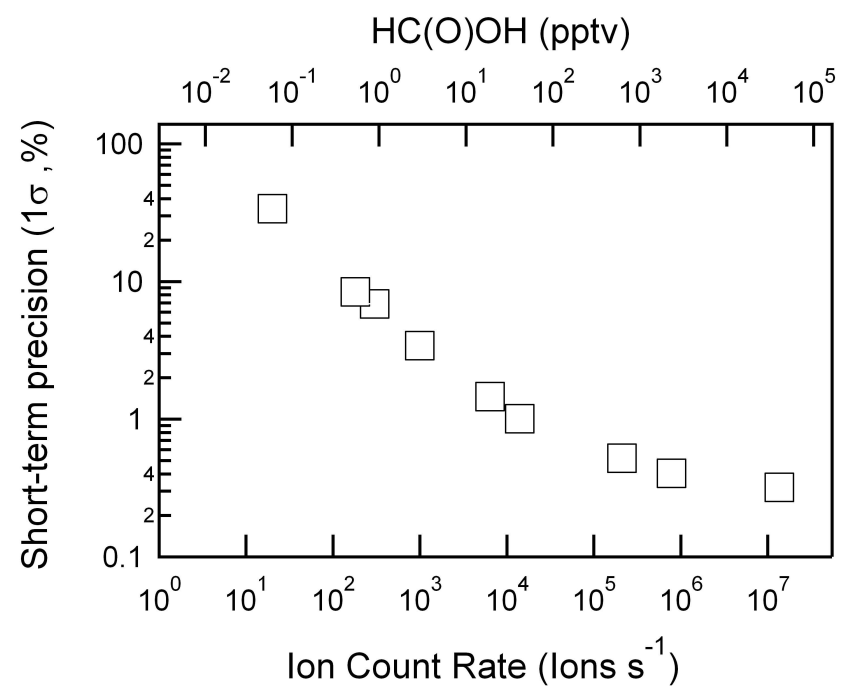

Fig. 7. Dependence of instrument short-term precision on the measured ion count rate.

(Fig. 7). As shown in Fig. 7, the $1 \sigma$ short-term precision in the CI-TOFMS is better than $5 \%$ for count rates associated with formic acid concentrations of 1 pptv or higher.

In theory, detection limits can be improved by signal averaging, where the signal-to-noise ratio increases as the square root of the integration time (Eq. 1). However, instrumental drift, in background or calibration factors, can often become significant on timescales shorter than the desired averaging time. The timescale for which averaging no longer improves signal-to-noise can be determined through calculation of the Allan variance (Werle et al., 1993). The Allan variance was calculated using the normalized count rate at $-45 \mathrm{Th}$ from 15 min of continuous sampling on a constant formic acid mixing ratio. Here, the count rate was normalized by the ratio of the reagent ion count rate in the absence of formic acid to that observed at this specific formic acid concentration. This analysis shows that the optimal integration time is 25 seconds, leading to a detection limit of $0.5 \mathrm{pptv}$ within $25 \mathrm{~s}$ (Fig. 8).

\section{Conclusions}

We report a new field-deployable, chemical ionization timeof-flight mass spectrometer. Instrument performance was assessed using acetate ion chemistry, with application to the selective detection of formic acid. The instrument as currently packaged is compact enough to be deployed to a broad range of sampling platforms, as demonstrated here through measurements obtained aboard the $R / V$ Atlantis off the coast of California. We show that the CI-TOFMS, as configured is sensitive ( $>300$ ions s$^{-1} \mathrm{pptv}^{-1}$ ) and precise ( $5 \%$ at $\left.1 \mathrm{pptv}\right)$, leading to a $3 \sigma$ detection limit of $4 \mathrm{pptv}$ ( $1 \mathrm{~s}$ averages). The detection limit of the current version of the CI-TOFMS is limited by the magnitude and variability in the background

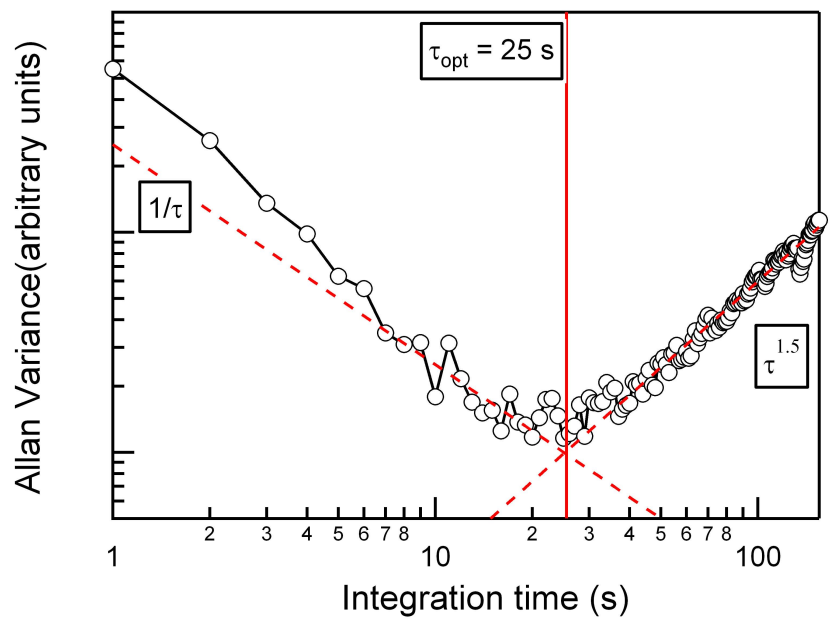

Fig. 8. Allan variance plot, calculated using the normalized count rate at $-45 \mathrm{Th}$, from $15 \mathrm{~min}$ of continuous $1 \mathrm{~Hz}$ sampling on the formic acid calibration standard. At low integration times, white noise is the dominate source of drift.

determination. The sensitivity, detection limit, and time response reported here, suggests that the CI-TOFMS will likely be capable of measuring trace gas fluxes of a host of compounds simultaneously via eddy correlation techniques. Application of CI-TOFMS to the detection of other inorganic and organic acids, as well as the use of different reagent ion molecules (e.g. $\mathrm{I}^{-}, \mathrm{CF}_{3} \mathrm{O}^{-}, \mathrm{CO}_{3}^{-}$) is promising, as we have demonstrated efficient transmission and detection of both bare ions and their associated ion-molecule clusters.

Acknowledgements. We thank J. L. Jimenez for sharing laboratory space during the testing of the initial CI-TOFMS prototype. Development efforts at ARI were supported by DOE and NOAA, grants DE-SC0004577 and NA09AR4310125, respectively.

Edited by: E. C. Apel

\section{References}

Berresheim, H., Elste, T., Plass-Dulmer, C., Eisele, F. L., and Tanner, D. J.: Chemical ionization mass spectrometer for long-term measurements of atmospheric $\mathrm{OH}$ and $\mathrm{H}_{2} \mathrm{SO}_{4}$, Int. J. Mass Spectrom., 202(1-3), 91-109, 2000.

Blake, R. S., Whyte, C., Hughes, C. O., Ellis, A. M., and Monks, P. S.: Demonstration of proton-transfer reaction time-of-flight mass spectrometry for real-time analysis of trace volatile organic compounds, Anal. Chem., 76(13), 3841-3845, 2004.

Blake, R. S., Wyche, K. P., Ellis, A. M., and Monks, P. S.: Chemical ionization reaction time-of-flight mass spectrometry: Multireagent analysis for determination of trace gas composition, Int. J. Mass Spectrom., 254(1-2), 85-93, 2006.

DeCarlo, P. F., Kimmel, J. R., Trimborn, A., Northway, M. J., Jayne, J. T., Aiken, A. C., Gonin, M., Fuhrer, K., Horvath, T., Docherty, K. S., Worsnop, D. R., and Jimenez, J. L.: Field-deployable, high-resolution, time-of-flight aerosol mass spectrometer, Anal. Chem., 78(24), 8281-8289, 2006. 
Ennis, C. J., Reynolds, J. C., Keely, B. J., and Carpenter, L. J.: A hollow cathode proton transfer reaction time of flight mass spectrometer, Int. J. Mass Spectrom., 247(1-3), 72-80, 2005.

Farmer, D. K. and Jimenez, J. L.: Real-time Atmospheric Chemistry Field Instrumentation, Anal. Chem., 82(19), 7879-7884, 2010.

Graul, S. T., Schnute, M. E., and Squires, R. R.: Gas-Phase Acidities of Carboxylic-Acids and Alcohols from Collision-Induced Dissociation of Dimer Cluster Ions, Int. J. Mass Spectrom., 96(2), 181-198, 1990.

Graus, M., Muller, M., and Hansel, A.: High Resolution PTR-TOF: Quantification and Formula Confirmation of VOC in Real Time, J. Am. Soc. Mass Spectrom., 21(6), 1037-1044, 2010.

Grosjean, D., Tuazon, E. C., and Fujita, E.: Ambient FormicAcid in Southern-California Air - a Comparison of 2 Methods, Fourier-Transform Infrared-Spectroscopy and Alkaline Trap Liquid-Chromatography with UV Detection, Environ. Sci. Technol., 24(1), 144-146, 1990.

Guilhaus, M., Selby, D., and Mlynski, V.: Orthogonal acceleration time-of-flight mass spectrometry, Mass Spectrom. Rev., 19(2), 65-107, 2000.

Hearn, J. D. and Smith, G. D.: A chemical ionization mass spectrometry method for the online analysis of organic aerosols, Anal. Chem., 76(10), 2820-2826, 2004.

Huey, L. G.: Measurement of trace atmospheric species by chemical ionization mass spectrometry: Speciation of reactive nitrogen and future directions, Mass Spectrom. Rev., 26(2), 166-184, 2007

Huey, L. G., Hanson, D. R., and Howard, C. J.: Reactions of $\mathrm{SF}_{6}^{-}$ and $\mathrm{I}^{-}$with Atmospheric Trace Gases, J. Phys. Chem., 99(14), 5001-5008, 1995.

Jordan, A., Haidacher, S., Hanel, G., Hartungen, E., Mark, L., Seehauser, H., Schottkowsky, R., Sulzer, P., and Mark, T. D.: A high resolution and high sensitivity proton-transfer-reaction time-of-flight mass spectrometer (PTR-TOF-MS), Int. J. Mass Spectrom., 286(2-3), 122-128, 2009.

Junninen, H., Ehn, M., Petäjä, T., Luosujärvi, L., Kotiaho, T., Kostiainen, R., Rohner, U., Gonin, M., Fuhrer, K., Kulmala, M., and Worsnop, D. R.: A high-resolution mass spectrometer to measure atmospheric ion composition, Atmos. Meas. Tech., 3, 10391053, doi:10.5194/amt-3-1039-2010, 2010.

Keene, W. C., Talbot, R. W., Andreae, M. O., Beecher, K., Berresheim, H., Castro, M., Farmer, J. C., Galloway, J. N., Hoffmann, M. R., Li, S. M., Maben, J. R., Munger, J. W., Norton, R. B., Pszenny, A. P., Puxbaum, H., Westberg, H., and Winiwarter, W.: An Intercomparison of Measurement Systems for Vapor and Particulate Phase Concentrations of Formic and Acetic-Acids, J. Geophys. Res.-Atmos., 94(D5), 6457-6471, 1989.

Kercher, J. P., Riedel, T. P., and Thornton, J. A.: Chlorine activation by $\mathrm{N}_{2} \mathrm{O}_{5}$ : simultaneous, in situ detection of $\mathrm{ClNO}_{2}$ and $\mathrm{N}_{2} \mathrm{O}_{5}$ by chemical ionization mass spectrometry, Atmos. Meas. Tech., 2, 193-204, doi:10.5194/amt-2-193-2009, 2009.

Mielke, L. H., Erickson, D. E., McLuckey, S. A., Muller, M., Wisthaler, A., Hansel, A., and Shepson, P. B.: Development of a Proton-Transfer Reaction-Linear Ion Trap Mass Spectrometer for Quantitative Determination of Volatile Organic Compounds, Anal. Chem., 80(21), 8171-8177, 2008.

Morrison, G. C. and Howard, C. J.: Selective detection of gas-phase aldehydes and ketones using protonated hydrazine, Int. J. Mass
Spectrom., 210(1-3), 503-509, 2001.

Müller, M., Mielke, L. H., Breitenlechner, M., McLuckey, S. A., Shepson, P. B., Wisthaler, A., and Hansel, A.: MS/MS studies for the selective detection of isomeric biogenic VOCs using a Townsend Discharge Triple Quadrupole Tandem MS and a PTR-Linear Ion Trap MS, Atmos. Meas. Tech., 2, 703-712, doi:10.5194/amt-2-703-2009, 2009.

Paulot, F., Wunch, D., Crounse, J. D., Toon, G. C., Millet, D. B., DeCarlo, P. F., Vigouroux, C., Deutscher, N. M., González Abad, G., Notholt, J., Warneke, T., Hannigan, J. W., Warneke, C., de Gouw, J. A., Dunlea, E. J., De Mazière, M., Griffith, D. W. T., Bernath, P., Jimenez, J. L., and Wennberg, P. O.: Importance of secondary sources in the atmospheric budgets of formic and acetic acids, Atmos. Chem. Phys., 11, 1989-2013, doi:10.5194/acp-11-1989-2011, 2011.

Prazeller, P., Palmer, P. T., Boscaini, E., Jobson, T., and Alexander, M.: Proton transfer reaction ion trap mass spectrometer, Rapid Commun. Mass Sp., 17(14), 1593-1599, 2003.

Roberts, J. M., Veres, P., Warneke, C., Neuman, J. A., Washenfelder, R. A., Brown, S. S., Baasandorj, M., Burkholder, J. B., Burling, I. R., Johnson, T. J., Yokelson, R. J., and de Gouw, J.: Measurement of HONO, HNCO, and other inorganic acids by negative-ion proton-transfer chemical-ionization mass spectrometry (NI-PT-CIMS): application to biomass burning emissions, Atmos. Meas. Tech., 3, 981-990, doi:10.5194/amt-3-981-2010, 2010.

Slusher, D. L., Pitteri, S. J., Haman, B. J., Tanner, D. J., and Huey, L. G.: A chemical ionization technique for measurement of pernitric acid in the upper troposphere and the polar boundary layer, Geophys. Res. Lett., 28(20), 3875-3878, 2001.

Steeghs, M. M. L., Sikkens, C., Crespo, E., Cristescu, S. M., and Harren, F. J. M.: Development of a proton-transfer reaction ion trap mass spectrometer: Online detection and analysis of volatile organic compounds, Int. J. Mass Spectrom., 262(1-2), 16-24, 2007.

Tanimoto, H., Aoki, N., Inomata, S., Hirokawa, J., and Sadanaga, Y.: Development of a PTR-TOFMS instrument for real-time measurements of volatile organic compounds in air, Int. J. Mass Spectrom., 263(1), 1-11, 2007.

Veres, P., Roberts, J. M., Warneke, C., Welsh-Bon, D., Zahniser, M., Herndon, S., Fall, R., and de Gouw, J.: Development of negativeion proton-transfer chemical-ionization mass spectrometry (NIPT-CIMS) for the measurement of gas-phase organic acids in the atmosphere, Int. J. Mass Spectrom., 274(1-3), 48-55, 2008.

Warneke, C., de Gouw, J. A., Lovejoy, E. R., Murphy, P. C., Kuster, W. C., and Fall, R.: Development of proton-transfer ion trap-mass spectrometry: On-line detection and identification of volatile organic compounds in air, J. Am. Soc. Mass Spectrom., 16(8), 1316-1324, 2005.

Werle, P., Mucke, R., and Slemr, F.: The Limits of Signal Averaging in Atmospheric Trace-Gas Monitoring by Tunable Diode-Laser Absorption-Spectroscopy (TDLAS), Appl. Phys. BPhoto., 57(2), 131-139, 1993.

Wyche, K. P., Blake, R. S., Ellis, A. M., Monks, P. S., Brauers, T., Koppmann, R., and Apel, E. C.: Technical Note: Performance of Chemical Ionization Reaction Time-of-Flight Mass Spectrometry (CIR-TOF-MS) for the measurement of atmospherically significant oxygenated volatile organic compounds, Atmos. Chem. Phys., 7, 609-620, doi:10.5194/acp-7-609-2007, 2007. 